Astronomy Letters, 2004, Vol. 30, No. 12, pp. 848-853.

\title{
ASTROMETRIC CONTROL OF THE INERTIALITY OF THE HIPPARCOS CATALOG
}

\author{
V.V. Bobylev \\ Pulkovo Astronomical Observatory, St. Petersburg, Russia
}

\begin{abstract}
Based on the most complete list of the results of an individual comparison of the proper motions for stars of various programs common to the Hipparcos catalog, each of which is an independent realization of the inertial reference frame with regard to stellar proper motions, we redetermined the vector $\boldsymbol{\omega}$ of residual rotation of the ICRS system relative to the extragalactic reference frame. The equatorial components of this vector were


$\omega_{z}=-0.35 \pm 0.09$ mas $\mathrm{yr}^{-1}$.
\end{abstract}

\section{INTRODUCTION}

A kinematic analysis performed previously (Bobylev 2004) revealed appreciable residual rotation of the stars of the Hipparcos catalog (ESA 1997) with respect to the inertial reference frame around the Galactic y axis with an angular velocity of $-0.36 \pm 0.09 \mathrm{mas} \mathrm{yr}^{-1}$ (milliarcseconds per year). A shortcoming of the method used is the absence of a rigorous criterion for separating the actual rotation of stars close to the Sun from the sought systematic rotation. The method of analyzing the proper-motion differences for stars of various programs common to the Hipparcos catalog, each of which is an independent realization of the inertial reference frame with regard to stellar proper motions, is free from this shortcoming. We call this method astrometric. Based on this method, Kovalevsky et al. (1997) found that all three components of the vector $\boldsymbol{\omega}$ of residual rotation of the Hipparcos catalog with respect to the extragalactic reference frame have no significant deviations from zero,

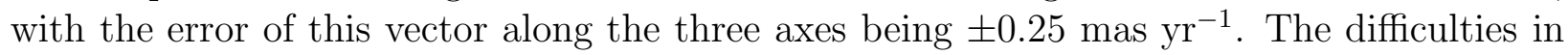
applying this method stem from the fact that there are few VLBI observable radio stars, while photographic catalogs are not free from the magnitude dependence of the stellar proper motions (the magnitude equation). The goal of this study is to redetermine the vector $\boldsymbol{\omega}$ using the most complete list of the independent results from an individual comparison of the stellar proper motions.

\section{CHARACTERISTIC OF INDIVIDUAL SOLUTIONS}

Based on the NPM photographic program of the Lick observatory (Klemola et al. 1987), two catalogs of absolute proper motions for northern-sky stars have been published: the NPM1 catalog of stars in 899 areas outside the Milky Way zone (Klemola et al. 1994) and the NPM2 catalog of stars in 347 areas of the Milky Way zone (Hanson et al. 2003). The areas of the NPM1 and NPM2 catalogs do not overlap. The results of a comparison of the proper motions for NPM1 and Hipparcos stars were described by Platais et al. (1998b) and designated as NPM (Yale) by Kovalevsky et al. (1997). An independent analysis of the 
Table 1: Components of the vector $\boldsymbol{\omega}-\omega_{x}, \omega_{y}$, and $\omega_{z}$ (in mas $\mathrm{yr}^{-1}$ ) that we determined from the VLA-Hipparcos differences using data from Boboltz et al. (2003).

\begin{tabular}{|l|c|ccc|}
\hline Number of stars & $\sigma_{\circ}$ & $\omega_{x}$ & $\omega_{y}$ & $\omega_{z}$ \\
\hline $15($ all $)$ & \pm 2.06 & $-0.97 \pm 0.62$ & $-1.59 \pm 0.73$ & $+0.18 \pm 0.63$ \\
$12\left(^{*}\right)$ & \pm 1.63 & $-0.42 \pm 0.56$ & $-0.51 \pm 0.64$ & $+0.20 \pm 0.57$ \\
\hline
\end{tabular}

(*) The radio stars HD $50896 \mathrm{~N}, \mathrm{KQ}$ Pup, and RS CVn were rejected.

proper-motion differences between the NPM1 and Hipparcos catalogs was also performed in Heidelberg; Kovalevsky et al. (1997) designated the results of this analysis as NPM (Heidelberg). All of the above authors agree that the component $\omega_{z}$ is difficult to determine using the NPM1 catalog, because there is a magnitude equation in the NPM1 proper motions

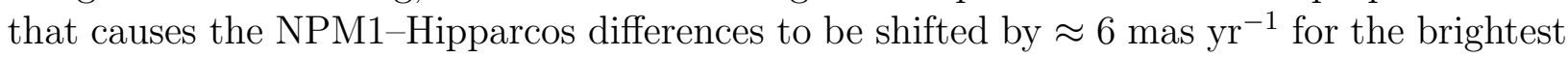
$\left(\approx 8^{m}\right)$ and faintest $\left(\approx 12^{m}\right)$ stars. Kovalevsky et al. (1997) did not include the component $\omega_{z}$ determined from NPM1 stars in their final solution.

Zhu (2003) compared the proper motions of NPM2 and Hipparcos stars. This author found no appreciable magnitude dependence of the NPM2-Hipparcos differences.

Based on the SPM program of photographic observations of southern-sky stars (Platais et al. 1995), Platais et al. (1998a) published the SPM2 catalog of absolute proper motions of stars in 156 southern sky areas. The results of a comparison of the absolute proper motions of SPM stars in 63 areas with the Hipparcos catalog were described by Platais et al. (1998a). We designate these SPM stars as SPM1. Zhu (2001) compared the proper motions of SPM2 and Hipparcos stars.

Based on the combined photographic catalog GPM (Rybka and Yatsenko 1997a), Rybka and Yatsenko (1997b) published a list of proper motions for bright stars common to the Hipparcos catalog designated as GPM1. Kislyuk et al. (1997) compared the proper motions of GPM1 and Hipparcos stars. These authors concluded that there is a magnitude equation in the GPM1 catalog, and the parameters $\omega_{x}, \omega_{y}$, and $\omega_{z}$ determined only from faint comparison stars must be used.

Bobylev et al. (2004) determined the parameters $\omega_{x}, \omega_{y}$, and $\omega_{z}$ by comparing the Pulkovo photographic catalog PUL2 and Hipparcos and found no appreciable magnitude equation in the proper motions of PUL2 stars. Each of the GPM and PUL2 catalogs is an independent realization of the photographic plan by Deutch (1954); the centers of the areas coincide and correspond to the list of Deutch (1955).

The results of the well-known independent programs were presented by Kovalevsky et al. (1997). In addition, the results of the Potsdam program were presented by Hirte et al. (1996), and the Bonn program by Geffert et al. (1997) and Tucholke et al. (1997); the Earth Rotation Parameters (EOP) were analyzed by Vondrak et al. (1997); radio stars were analyzed by Lestrade et al. (1995), and the Hubble Space Telescope (HST) observations were analyzed by Kovalevsky et al. (1997).

The main difference between our approach and the approach of Kovalevsky et al. (1997) is that we included the following data:

(1) The results of a comparison of the proper motions for PUL2 and Hipparcos stars (Bobylev et al. 2004); 
(2) The results of a comparison of the proper motions for SPM2 and Hipparcos stars (Zhu 2001);

(3) The results of a comparison of the proper motions for NPM2 and Hipparcos stars (Zhu 2003);

(4) The results of a comparison of the VLA absolute proper motions for radio stars and their Hipparcos proper motions.

Table 1 gives the components of the vector $\boldsymbol{\omega}$ that we calculated using the absolute proper motions of 15 radio stars from Boboltz et al. (2003). These authors described in detail the project and the method of referencing the observations of radio stars to quasars and compared these stars with the Hipparcos catalog, but did not determine the components of the vector $\boldsymbol{\omega}$. We used the equations in the form that was proposed and used by Lindegren and Kovalevsky (1995):

$$
\begin{gathered}
\Delta \mu_{\alpha} \cos \delta=\omega_{x} \cos \alpha \sin \delta+\omega_{y} \sin \alpha \sin \delta-\omega_{z} \cos \delta \\
\Delta \mu_{\delta}=-\omega_{x} \sin \alpha+\omega_{y} \cos \alpha
\end{gathered}
$$

where the Hipparcos catalog differences are on the left-hand sides of the equations. Here, $\sigma_{\circ}$ is the error per unit weight in the solution of Eqs. (1) and (2). The VLA (Boboltz et al. 2003) and VLBI (Lestrade et al. 1995, 1999) absolute proper motions are independent.

Our approach also differs from that of Kovalevsky et al. (1997) in that we used the results of a comparison of the NPM1 and Hipparcos stellar proper motions performed by the Heidelberg team, which were obtained from the data for 2616 stars, to determine $\omega_{z}$. The random errors in $\omega_{x}, \omega_{y}$, and $\omega_{z}$ for the Heidelberg solution are given only for the sample of

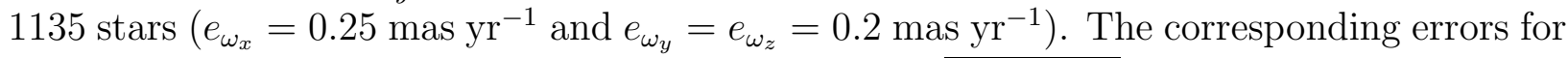
the sample of 2616 stars are smaller by a factor of $\approx \sqrt{2616 / 1135}$. Since there are problems with the NPM1 catalog, we assumed the random errors to be $0.25 \mathrm{mas} \mathrm{yr}^{-1}$ for $\omega_{x}$ and 0.20 mas $\mathrm{yr}^{-1}$ for $\omega_{y}$ and $\omega_{z}$ in order not to overestimate this solution. Our choice of this solution was dictated by the fact that it was obtained in the magnitude range $10.5^{m}-11.5^{m}$. As can be seen in Fig. 1c from Platais et al. (1998b), the Hipparcos-NPM1 proper-motion differences have a horizontal pattern near zero precisely in this magnitude range. In our opinion, the NPM1 proper motions are least affected by the magnitude equation in this magnitude range. In addition, we used only one of the available (not independent) results of a comparison of the SPM1 and Hipparcos catalogs, the YVA solution (Kovalevsky et al. 1997; Platais et al. 1998b).

\section{DETERMINING THE VECTOR $\boldsymbol{\omega}$}

We assigned a weight inversely proportional to the square of the error e in the corresponding quantities $\omega_{x}, \omega_{y}$, and $\omega_{z}$ to each comparison catalog, which was calculated using the formula

$$
P_{i}=e_{k i e v}^{2} / e_{i}^{2}, \quad i=1, \ldots, 12,
$$

where $i$ is the number of individual sources, and $e_{k i e v}$, the random error of the GPM1 (Kiev) program. Table 2 contains the data used here. The second column of the table gives the weights calculated using formula (3). Not all of the authors use equations in the form (1) and (2). In such cases, we reduced the signs of the quoted quantities (Zhu 2001, 2003) to the same form. 
Table 2: (Equatorial) components of the vector of residual rotation of the Hipparcos catalog with respect to extragalactic objects $-\omega_{x}, \omega_{y}$, and $\omega_{z}\left(\right.$ mas $\left.\mathrm{yr}^{-1}\right)$.

\begin{tabular}{|c|c|c|c|c|c|c|}
\hline & $P_{x}, P_{y}, P_{z}$ & $N_{\star}$ & $N_{\text {area }}$ & $\omega_{x}$ & $\omega_{y}$ & $\omega_{z}$ \\
\hline NPM2 & $16.00 / 4.62 / 8.16$ & 3519 & 347 & $-0.11 \pm 0.20$ & $-0.19 \pm 0.20$ & $-0.75 \pm 0.28$ \\
\hline SPM2 & $22.15 / 9.43 / 28.44$ & 9356 & 156 & $+0.10 \pm 0.17$ & $+0.48 \pm 0.14$ & $-0.17 \pm 0.15$ \\
\hline NPM1 & $10.24 / 4.62 / 16.00$ & 2616 & 899 & $-0.76 \pm 0.25$ & $+0.17 \pm 0.20$ & $-0.85 \pm 0.20$ \\
\hline SPM1 & $44.44 / 5.71 / 130.61$ & 4067 & 63 & $+0.44 \pm 0.12$ & $+0.71 \pm 0.18$ & $-0.30 \pm 0.07$ \\
\hline PUL2 & $2.90 / 1.28 / 3.63$ & 1004 & 147 & $-0.98 \pm 0.47$ & $-0.03 \pm 0.38$ & $-1.66 \pm 0.42$ \\
\hline $\operatorname{Kiev}(\mathrm{GPM} 1)$ & $1.0 / 1.0 / 1.0$ & 415 & 154 & $-0.27 \pm 0.80$ & $+0.15 \pm 0.60$ & $-1.07 \pm 0.80$ \\
\hline Potsdam & $2.37 / 0.74 / 2.78$ & 256 & 24 & $+0.22 \pm 0.52$ & $+0.43 \pm 0.50$ & $+0.13 \pm 0.48$ \\
\hline Bonn & $5.54 / 2.96 / 5.88$ & 88 & 13 & $+0.16 \pm 0.34$ & $-0.32 \pm 0.25$ & $+0.17 \pm 0.33$ \\
\hline VLBI & $7.11 / 2.74 / 7.11$ & 12 & & $-0.16 \pm 0.30$ & $-0.17 \pm 0.26$ & $-0.33 \pm 0.30$ \\
\hline $\mathrm{VLA}+\mathrm{PT}$ & $2.04 / 0.45 / 1.97$ & 12 & & $-0.42 \pm 0.56$ & $-0.51 \pm 0.64$ & $+0.20 \pm 0.57$ \\
\hline HST & $0.08 / 0.08 / 0.05$ & 46 & & $-1.60 \pm 2.87$ & $-1.92 \pm 1.54$ & $+2.26 \pm 3.42$ \\
\hline $\mathrm{EOP}$ & $8.16 / 2.36 /-$ & & & $-0.93 \pm 0.28$ & $-0.32 \pm 0.28$ & - \\
\hline $\begin{array}{l}\text { Mean } 1 \\
\text { Mean } 2\end{array}$ & & & & $\begin{array}{l}+0.04 \pm 0.15 \\
-0.19 \pm 0.16\end{array}$ & $\begin{array}{l}+0.18 \pm 0.12 \\
+0.08 \pm 0.11\end{array}$ & $\begin{array}{l}-0.35 \pm 0.09 \\
-0.43 \pm 0.15\end{array}$ \\
\hline
\end{tabular}

Note: $N_{\star}$ is the number of common comparison stars, and $N_{\text {area }}$ is the number of comparison areas common to the photographic catalogs. Mean 2 was calculated without SPM1.

The lower part of Table 2 gives the weighted means of $\omega_{x}, \omega_{y}$, and $\omega_{z}$. Mean 1 was calculated using all of the available data in Table 2. The SPM1 and SPM2 catalogs are not independent, but the methods of obtaining the solutions differ. The SPM1 solution (Platais et al. 1998b) was obtained only from the differences in $\mu_{\alpha} \cos \delta$ (e.g., only from Eq. (1)). The SPM2 solution (Zhu 2001) was obtained by simultaneously solving Eqs. (1) and (2); the author pointed out that there is a color equation in the SPM2 catalog, which was not eliminated, and it is more pronounced in the $\mu_{\delta}$ differences. We calculated Mean 2 without using the SPM1 solution in order to analyze only independent sources. In this case, the SPM proper motions also have the largest weight when calculating the component $\omega_{z}$, as can be seen from the table. A comparison of Means 1 and 2 reveals no significant differences between these two solutions, with $\omega_{z}$ being an appreciable component. The errors of the vector $\boldsymbol{\omega}$ along the three axes $e_{\omega}=\sqrt{{e_{\omega_{x}}}^{2}+e_{\omega_{y}}{ }^{2}+e_{\omega_{z}}{ }^{2}}$ are \pm 0.21 and $\pm 0.25 \mathrm{mas} \mathrm{yr}^{-1}$ for Mean 1 and 2 solutions, respectively. Our solutions for the components $\omega_{x}$ and $\omega_{y}$ do not differ significantly from the final solutions of Kovalevsky et al. (1997) based on both Lindegren's and Kovalevsky's methods. At the same time, there is a significant difference in the determination of $\omega_{z}$, which we found to differ significantly from zero. The figure shows the projections of the individual solutions for the vector $\boldsymbol{\omega}$ onto the $x y, x z$, and $y z$ planes based on the data from Table 2. Also shown in the figure are the components of our Mean 1 solution.

\section{DISCUSSION}

The effects of the actual motions of stars in the differences between the catalogs under 
consideration are ruled out. Therefore, the causes of the rotation $\omega_{z}$ found are the following:

(1) Inaccurate realization of the ICRS or, in other words, residual rotation of the Hipparcos catalog with respect to the ICRF (Ma et al. 1998), and, in this case, the rotation found is of a "technical" nature;

(2) Residual rotation of the ICRF itself with respect to the extragalactic reference frame. The ICRF is based on ground-based VLBI observations of extragalactic radio sources. It may be assumed that, in this case, the rotation $\omega_{z}$ found is precessional in nature and depends on the accuracy of the constants used. Given the form of the proper-motion differences and the signs on the right-hand sides of Eqs. (1)-(2), we can write

$$
\begin{gathered}
\omega_{y}=-\Delta p_{1} \sin \varepsilon, \\
\omega_{z}=\Delta p_{1} \cos \varepsilon-\Delta E,
\end{gathered}
$$

where $\Delta p_{1}$ is the correction to the adopted constant of lunisolar precession in longitude, $\Delta E$ is the sum of the corrections to the rate of planetary precession and the motion of the zero point of right ascensions, and $\varepsilon$ is the inclination of the ecliptic to the equator. Using only Eq. (5) and setting $\Delta E=0$, we obtain from our Mean 1 solution

$$
\Delta p_{1}=-0.38 \pm 0.10 \text { mas } \mathrm{yr}^{-1} .
$$

This value agrees with the result of our previous work (Bobylev 2004), where we found $\Delta p_{1}=-0.42 \pm 0.10$ mas $\mathrm{yr}^{-1}$ from a kinematic analysis of the proper motions for distant Hipparcos stars. In our opinion, a comparison of the residual rotations around the ecliptic axis found by two independent methods is most justifiable.

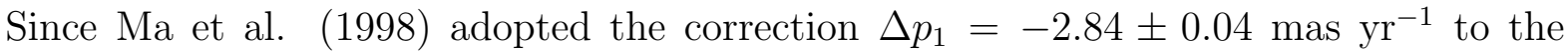
IAU (1976) constant of lunisolar precession in longitude, it may be assumed that $\Delta p_{1}$ was underestimated. Since the ICRS was constructed with precisely this value, solution (6) yields an "addition" to this correction. In this case, the correction to the IAU (1976) constant of lunisolar precession in longitude is

$$
\Delta p_{1}=-3.22 \pm 0.11 \text { mas } \mathrm{yr}^{-1} .
$$

Solution (7) is in satisfactory agreement with the most recent results from the laser ranging of the Moon, $\Delta p_{1}=-3.02 \pm 0.03$ mas yr$^{-1}$ (Chapront et al. 2002), and with the results of an analysis of radio interferometric observations, $\Delta p_{1}=-3.011 \pm 0.003 \mathrm{mas} \mathrm{yr}^{-1}$ (Fukushima 2003). On the other hand, if we assume that the lunisolar precession has no effect on the construction of ICRS and ICRF, then $\Delta E \neq 0$. In this case, we find from Eq. (5) that

$$
\Delta E=+0.38 \pm 0.10 \text { mas } \mathrm{yr}^{-1} .
$$

The residual rotation of the Hipparcos catalog that we found is small. An analysis of the radio-interferometric observations (Boboltz et al. 2003) performed 9.69 years after (epoch 2000.94) the construction of ICRS (epoch 1991.25) revealed no significant effect in the coordinates of radio stars. However, the rotation components obtained by Boboltz et al. (2003) from the coordinate differences for 18 radio stars (Kovalevsky et al. 1997) are of considerable interest:

$$
\begin{aligned}
& \varepsilon_{0 x}=+0.2 \pm 2.9 \mathrm{mas} \\
& \varepsilon_{0 y}=+1.9 \pm 3.2 \mathrm{mas} \\
& \varepsilon_{0 z}=-2.3 \pm 2.8 \mathrm{mas}
\end{aligned}
$$



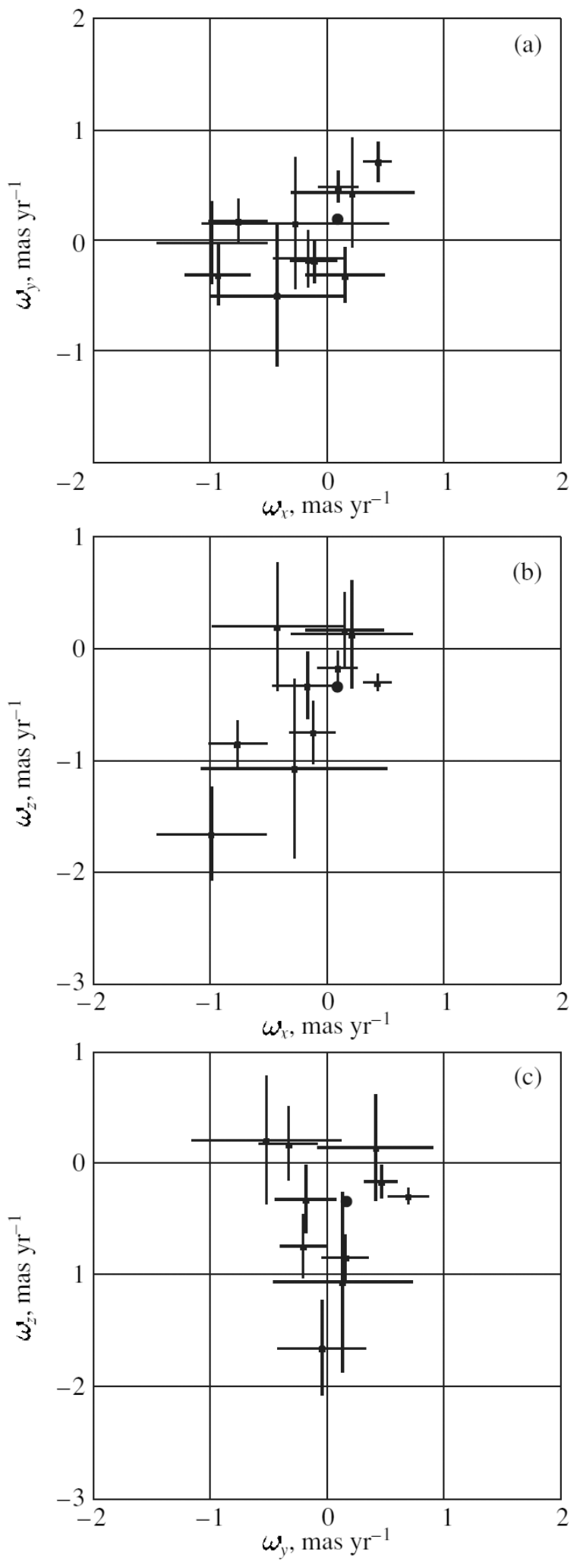

Figure 1: Projections of the individual solutions (without HST) for the vector $\boldsymbol{\omega}$ : (a) onto the $x y$ plane; (b) onto the $x z$ plane, and (c) onto the $y z$ plane. The filled circle indicates the components of our Mean 1 solution. 
We changed the signs of the components of the vector $\varepsilon$ inferred by Boboltz et al. (2003) in order to have Hipparcos catalog differences comparable with those analyzed. To compare results (9)-(11), for example, with the Mean 1 solution, the quantities $\varepsilon_{0 x, y, z}$ must be divided by the epoch difference. Thus, the difference $\varepsilon_{0 z}$, which is determined with the smallest random error, is largest among quantities (9)-(11), thereby confirming our $\omega_{z}$ value. In general, quantities (9)-(11) agree well with the Mean 1 solution.

Our analysis of the available individual sources for controlling the ICRS inertiality shows that they are of little use for analyzing future projects, such as GAIA, SIM, etc., in which a microacrsecond accuracy is expected to be reached (Kovalevsky et al. 1999). The idea that the inertiality must be controlled using quasar observations from a spacecraft directly during its flight (Metz and Geffert 2004) seems most promising.

\section{CONCLUSIONS}

We have confirmed that the error in referring the ICRS to the inertial reference frame is

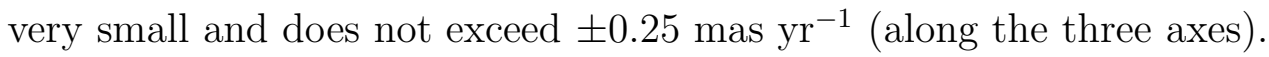

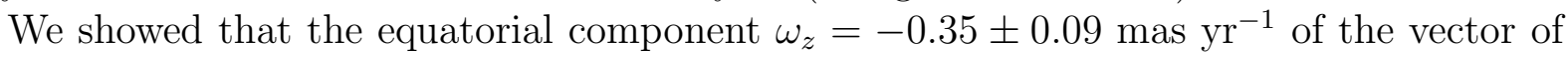
residual rotation of the ICRS with respect to the inertial reference frame differs significantly from zero. This confirms the result of a kinematic analysis of the proper motions for stars of the ICRS catalogs (Bobylev 2004).

\section{ACKNOWLEDGMENTS}

This work was supported by the Russian Foundation for Basic Research (project no. 02-02-16570).

\section{REFERENCES}

1. D.A. Boboltz, A.L. Fey, K.J. Johnston, et al., Astron. J. 126, 484 (2003).

2. V.V. Bobylev, Pis'ma Astron. Zh. 30, 289 (2004) [Astron. Lett. 30, 251 (2004)].

3. V.V. Bobylev, N. M. Bronnikova, and N. A. Shakht, Pis'ma Astron. Zh. 30, 519 (2004).

4. J. Chapront, M. Chapront-Touzé, and G. Francou, Astron. Astrophys. 387, 700 (2002).

5. A.N. Deutch, Trans. IAU 8, 789 (1954).

6. A.N. Deutch, V.V. Lavdovskii, and N.V. Fatchikhin, Izv. Gos. Astron. Obs. 154, 14 (1955).

7. T. Fukushima, Astron. J. 126, 494 (2003).

8. M. Geffert, A. R. Klemola, M. Hiesgen, et al., Astron. Astrophys. 124, 157 (1997).

9. R.B. Hanson, A.R. Klemola, B.F. Jones, et al., Lick Northern Proper Motion Program: NPM2 Catalog, http://www.ucolick.org/?npm/NPM2/ (2003).

10. The HIPPARCOS and Tycho Catalogues, ESA SP- 1200 (ESA, 1997).

11. S. Hirte, E. Schilbach, and R.-D. Scholz, Astron. Astrophys., Suppl. Ser. 126, 31 (1996).

12. V.S. Kislyuk, S.P. Rybka, A.I. Yatsenko, et al., Astron. Astrophys. 321, 660 (1997).

13. A.R. Klemola, R.B.Hanson, and B.F. Jones, Galactic and Solar System Optical Astrometry, Ed. by L.V. Morrison and G.F. Gilmore (Cambridge Univ. Press, Cambridge, 1994), p. 20.

14. A.R. Klemola, B.F. Jones, and R.B.Hanson, Astron. J. 94, 501 (1987).

15. J. Kovalevsky, JOURNEÉS 1999, Ed. by M. Soffel and N. Capitaine (Obs. de Paris, Paris, 1999), p. 103.

16. J. Kovalevsky, L. Lindegren, M.A.C. Perryman, et al., Astron. Astrophys. 323, 620 (1997).

17. J.-F. Lestrade, D.L. Jones, R.A. Preston, et al., Astron. Astrophys. 304, 182 (1995).

18. J.-F. Lestrade, R. A. Preston, D.I. Jones, et al., Astron. Astrophys. 344, 1014 (1999).

19. L. Lindegren and J. Kovalevsky, Astron. Astrophys. 304, 189 (1995). 
20. C. Ma, E.F. Arias, T.M. Eubanks, et al., Astron. J. 116, 516 (1998).

21. M. Metz and M. Geffert, Astron. Astrophys. 413, 771 (2004).

22. I. Platais, T.M. Girard, V. Kozhurina-Platais, et al., Astron. J. 116, 2556 (1998a).

23. I. Platais, T.M. Girard, W.F. van Altena, et al., Astron. Astrophys. 304, 141 (1995).

24. I. Platais, V. Kozhurina-Platais, T.M. Girard, et al., Astron. Astrophys. 331, 1119 (1998b).

25. S.P. Rybka and A.I. Yatsenko, Kinemat. Fiz. Neb. Tel 13, 70 (1997a).

26. S.P. Rybka and A.I. Yatsenko, Astron. Astrophys., Suppl. Ser. 121, 243 (1997b).

27. H.-J. Tucholke, P. Brosche, and M. Odenkirchen, Astron. Astrophys., Suppl. Ser. 124, 157 (1997).

28. J. Vondrák, C. Ron, and I. Pešek, Astron. Astrophys. 319, 1020 (1997).

29. Zi Zhu, Publ. Astron. Soc. Jpn. 53, L33 (2001).

30. Zi Zhu, JOURNEÉS 2003, Ed. N. Capitaine (Obs. de Paris, Paris, 2003). 